\title{
MASSES, LUMINOSITIES, AND ORBITAL COPLANARITIES OF THE $\mu$ ORIONIS QUADRUPLE-STAR SYSTEM FROM PHASES DIFFERENTIAL ASTROMETRY
}

\author{
Matthew W. Muterspaugh ${ }^{1}$, Benjamin F. Lane ${ }^{2}$, Francis C. Fekel $^{3}$, Maciej Konacki ${ }^{4}$, Bernard F. Burke ${ }^{5}$, \\ S. R. KulKarni ${ }^{6}$, M. M. Colavita ${ }^{7}$, M. ShaO ${ }^{7}$, and Sloane J. WikTorowicz ${ }^{8}$ \\ ${ }^{1}$ Townes Fellow, University of California, Space Sciences Laboratory, 7 Gauss Way, Berkeley, CA 94720-7450, USA; matthew1@ ssl.berkeley.edu \\ ${ }^{2}$ Draper Laboratory, 555 Technology Square, Cambridge, MA 02139-3563, USA; blane@mit.edu \\ ${ }^{3}$ Tennessee State University, Center of Excellence in Information Systems, 3500 John A. Merritt Blvd., Box No. 9501, Nashville, TN 37203-3401, USA \\ ${ }^{4}$ Nicolaus Copernicus Astronomical Center, Polish Academy of Sciences, Rabianska 8, 87-100 Torun, Poland; maciej@ncac.torun.pl \\ ${ }^{5}$ MIT Kavli Institute for Astrophysics and Space Research, MIT Department of Physics, 70 Vassar Street, Cambridge, MA 02139, USA \\ ${ }^{6}$ Division of Physics, Mathematics and Astronomy, 105-24, California Institute of Technology, Pasadena, CA 91125, USA \\ ${ }^{7}$ Jet Propulsion Laboratory, California Institute of Technology, 4800 Oak Grove Dr., Pasadena, CA 91109, USA \\ ${ }^{8}$ Department of Geological and Planetary Sciences, California Institute of Technology, Pasadena, CA 91125, USA \\ Received 2007 July 9; accepted 2007 October 10; published 2008 January 29
}

\begin{abstract}
$\mu$ Orionis was identified by spectroscopic studies as a quadruple-star system. Seventeen high-precision differential astrometry measurements of $\mu$ Ori have been collected by the Palomar High-precision Astrometric Search for Exoplanet Systems (PHASES). These show both the motion of the long-period binary orbit and short-period perturbations superimposed on that caused by each of the components in the long-period system being themselves binaries. The new measurements enable the orientations of the long-period binary and short-period subsystems to be determined. Recent theoretical work predicts the distribution of relative inclinations between inner and outer orbits of hierarchical systems to peak near 40 and 140 degrees. The degree of coplanarity of this complex system is determined, and the angle between the planes of the A-B and Aa-Ab orbits is found to be $136.7 \pm 8.3$ degrees, near the predicted distribution peak at 140 degrees; this result is discussed in the context of the handful of systems with established mutual inclinations. The system distance and masses for each component are obtained from a combined fit of the PHASES astrometry and archival radial velocity observations. The component masses have relative precisions of 5\% (component Aa), 15\% (Ab), and $1.4 \%$ (each of $\mathrm{Ba}$ and $\mathrm{Bb}$ ). The median size of the minor axes of the uncertainty ellipses for the new measurements is 20 micro-arcseconds ( $\mu$ as). Updated orbits for $\delta$ Equulei, $\kappa$ Pegasi, and V819 Herculis are also presented.
\end{abstract}

Key words: astrometry - binaries: close - binaries: visual - stars: distances - stars: individual ( $\delta$ Equ, $\kappa$ Peg, $\mu$ Orionis, V819 Her) - techniques: interferometric

\section{INTRODUCTION}

$\mu$ Orionis (61 Ori, HR 2124, HIP 28614, and HD 40932) is a quadruple-star system that has been extensively studied by radial velocity (RV) and differential astrometry. It is located just north of Betelgeuse, Orion's right shoulder (left on the sky); $\mu$ Ori is a bright star that is visible to the unaided eye even in moderately light-polluted skies. Frost (1906) discovered it to be a short-period (4.5 days) single-lined spectroscopic binary; this was component $\mathrm{Aa}$, whose short-period, low-mass companion $\mathrm{Ab}$ has never been detected directly. Aitken (1914) discovered it also had a more distant component (B) forming a sub-arcsecond visual binary. Much later, Fekel (1980) found B was itself a short-period (4.78 days) double-lined spectroscopic binary, making the system quadruple; these stars are designated $\mathrm{Ba}$ and Bb. Most recently, Fekel et al. (2002; hereafter F2002) reported the astrometric orbit of the A-B motion, double-lined RV orbits for $\mathrm{A}-\mathrm{B}$ and the $\mathrm{Ba}-\mathrm{Bb}$ subsystem, and a single-lined RV orbit for the $\mathrm{Aa}-\mathrm{Ab}$ subsystem. F2002 estimate the spectral types as A5V (Aa, an Am star), F5V (Ba), and F5V (Bb), though they note that these classifications are less certain than usual due to the complexity of the system. For a more complete discussion of the history of $\mu$ Ori, see F2002.

Until now, astrometric observations have only been able to characterize the long-period $\mathrm{A}-\mathrm{B}$ motion, lacking the precision necessary to measure the astrometric perturbations to this orbit caused by the $\mathrm{Aa}-\mathrm{Ab}$ and $\mathrm{Ba}-\mathrm{Bb}$ subsystems. The method described by Lane \& Muterspaugh (2004) for ground-based differential astrometry at the $\sim 20 \mu$ as level for sub-arcsecond ("speckle") binaries has been used to study $\mu$ Ori during the 2004-2007 observing seasons. These measurements represent an improvement in precision of over two orders of magnitude over previous work on this system.

The goal of the current investigation is to report the center-oflight (photocenter) astrometric orbits of the $\mathrm{Aa}-\mathrm{Ab}$ and $\mathrm{Ba}-\mathrm{Bb}$ subsystems. This enables measurement of the coplanarities of the $\mathrm{A}-\mathrm{B}, \mathrm{Aa}-\mathrm{Ab}$, and $\mathrm{Ba}-\mathrm{Bb}$ orbits. The masses and luminosity ratio of $\mathrm{Aa}$ and $\mathrm{Ab}$ are measured for the first time. Also presented are updated orbits for the PHASES targets $\delta \mathrm{Equ}, \kappa \mathrm{Peg}$, and V819 Her.

Astrometric measurements were made at the Palomar Testbed Interferometer (PTI; Colavita et al. 1999) as part of the Palomar High-precision Astrometric Search for Exoplanet Systems (PHASES) program (Muterspaugh et al. 2006c). PTI is located on Palomar Mountain near San Diego, CA. It was developed by the Jet Propulsion Laboratory, California Institute of Technology for NASA, as a testbed for interferometric techniques applicable to the Keck Interferometer and other missions such as the Space Interferometry Mission (SIM). It operates in the $J(1.2 \mu \mathrm{m}), H(1.6 \mu \mathrm{m})$, and $K(2.2 \mu \mathrm{m})$ bands, and combines starlight from two out of three available $40 \mathrm{~cm}$ apertures. The apertures form a triangle with one 110 and two $87 \mathrm{~m}$ baselines. 


\section{OBSERVATIONS AND DATA PROCESSING}

\subsection{PHASES Observations}

\subsubsection{Instrumental Setup}

$\mu$ Ori was observed with PTI on 17 nights in 2004-2007 with the observing mode described in Lane \& Muterspaugh (2004). Starlight is collected from two apertures, collimated, and sent to a central beam combining facility. There, the light from each telescope reflects from movable mirrors (delay lines) whose position is constantly varied to account for sidereal motion and to track atmospheric index of refraction variations. After this first set of delay lines, a beamsplitter is used to divide the light from each telescope; $\sim 70 \%$ of the light is sent to an interferometric beam combiner that monitors a single fringe from any star in the field at rapid (10-20 ms) time scales to measure fringe phase variations caused by the atmosphere, and provide feedback to the main delay lines. This process phase-stabilizes the other $\sim 30 \%$ of the light (a technique known as phase referencing, Lane \& Colavita 2003), which is sent to a second interferometric beam combiner that can add an additional variable delay of order $250 \mu \mathrm{m}$ to the light from one telescope. This variable delay is modulated with a triangle waveform, scanning through interferograms from all stars within the sub-arcsecond field of view. These interferogram scans are the observables used for PHASES astrometry.

\subsubsection{Data Reduction}

Modifications to the data processing algorithm since the original report are given by Muterspaugh et al. (2005) and have been incorporated in the current study. Interferogram templates are fit to each scan, forming a likelihood function of the separations of interferograms formed by components $\mathrm{A}$ and $\mathrm{B}$. A grid of differential right ascension and declination is formed, and a $\chi^{2}$ likelihood surface is mapped onto this grid by converting delay separation to differential right ascension and declination. That $\chi^{2}$ surface is co-added over all the scans of $\mu$ Ori within the night (typically $\sim 1000$ scans or more). The deepest minimum in the $\chi^{2}$ surface corresponds to the binary separation, while the width of that minimum determines the uncertainty ellipse. Due to the oscillatory nature of the interferograms, other local minima can exist; these "sidelobes" are separated by the interferometer's resolution $\sim \lambda / B \sim 4$ mas ( $B$ is the separation between the telescopes, and $\lambda$ is the wavelength of starlight), an amount much larger than the width of an individual minimum. The signal-to-noise ratio $(\mathrm{S} / \mathrm{N})$ can be increased by co-adding many scans and by earth-rotation synthesis, which smears all but the true minimum, and the true minimum then can be established. Only those measurements for which no sidelobes appear at the $4 \sigma$ contour of the deepest minimum are used in orbit fitting.

All measurements have been processed using this new data reduction pipeline. The measurements are listed in Table 1 , in the ICRS 2000.0 reference frame.

\subsubsection{Technique Upgrades}

Data since mid-2006 have benefited from the use of an automatic alignment system and longitudinal dispersion compensator; the affected data points are noted in Table 1. These modifications reduced the throughput of the astrometry setup; to compensate, a $50 \mathrm{~Hz}$ phase-tracking rate was sometimes used, whereas observations previous to these changes utilized $100 \mathrm{~Hz}$ tracking for monitoring the atmosphere.
Drifts in optical alignment may result in variable pupil sampling at the interferometer apertures, changing the effective interferometric baseline. To minimize this potential systematic error, a continuous realignment system has been developed. A red laser is co-aligned with the starlight and reverse propagated through the interferometer. Four percent reflective pellicle beamsplitters are placed near the focuses of the interferometer telescopes to extract this tracer beam and redirect to a camera where the pupil is re-imaged. The angles of the first flat mirrors receiving incoming starlight in the beam-combining lab are continually adjusted by a closed-loop feedback system to hold the laser spot on the camera at the telescope.

The path compensation for the geometric delay at PTI has been done with movable mirrors in air, which has a wavelength-dependent index of refraction. The fringe packets of astrophysical sources are dispersed by an amount that depends on the difference in air paths between arms of the interferometer; this changes the shape and overall location of the fringe packets. To compensate, two prisms are introduced in each of the interferometer's two arms. The set in one arm is static. In the other pair, the prisms are slid relative to each other along their slope to introduce a variable amount of glass dispersion whose shape is opposite that of air to high order. This flattens the variability of delay versus wavelength. The system is calibrated by setting the telescope siderostats into a retroreflecting mode, using an internal white-light source to form interferograms, which are detected with a low-resolution spectrometer (five elements across the $K$ band), and measuring the offsets between the interferograms as a function of prism location. During observations, the prism position is continuously changed with an open-loop control calculated from the locations of the delay lines.

There are insufficient new data to establish the degree to which these instrumental changes might be reducing excess data scatter, or to establish a relative weighting between data subsets. No large discontinuities in the orbital motions are seen between pre- and post-upgrade subsets for $\mu$ Ori or the other PHASES targets, suggesting that the subsets are compatible. For the purposes of the current investigation, the PHASES observations are treated as a single data set with equal weighting on observations from before and after these upgrades.

\subsubsection{The PHASES Astrometric Orbits}

The differential astrometry measurements are listed in Table 1, in the ICRS 2000.0 reference frame. The existence of data scatter beyond the level estimated by formal uncertainties from the PHASES analysis algorithm was determined by model fitting the PHASES data alone. Model fitting was performed with standard $\chi^{2}$ sum of squared residuals minimization, slightly complicated by the two-dimensional nature of the uncertainty ellipses but still straightforward to carry out. The limited number and time span of the PHASES observations prevent an independent fitting of that data set to a four-body, 3-Keplerian model to determine potential noise excess. Thus the A-B period and $\mathrm{Aa}-\mathrm{Ab}$ and $\mathrm{Ba}-\mathrm{Bb}$ periods, eccentricities, and angles of periastron passages were fixed at the values reported in F2002 (in the case of $\mathrm{Ba}-\mathrm{Bb}$, which had zero eccentricity in F2002, the angle of periastron passage is undefined and fixed at zero). The minimum $\chi^{2}$ does not equal the number of degrees of freedom. An excess noise factor of 1.73 is found, and the PHASES uncertainties reported in Table 1 have been increased by this 
Table 1

PHASES Data for $\mu$ Ori

\begin{tabular}{|c|c|c|c|c|c|c|c|c|c|c|c|c|}
\hline HJD-2400000.5 & $\begin{array}{l}\delta \text { R.A. } \\
\text { (mas) }\end{array}$ & $\begin{array}{r}\delta \text { Decl. } \\
\text { (mas) }\end{array}$ & $\begin{array}{l}\sigma_{\min } \\
(\mu \text { as })\end{array}$ & $\begin{array}{l}\sigma_{\text {maj }} \\
(\mu \text { as })\end{array}$ & $\begin{array}{c}\phi_{\mathrm{e}} \\
(\mathrm{deg})\end{array}$ & $\begin{array}{c}\sigma_{\text {R.A. }} \\
(\mu \text { as })\end{array}$ & $\begin{array}{l}\sigma_{\text {Decl. }} \\
(\mu \text { as })\end{array}$ & $\frac{\sigma_{\text {R.A.,Decl. }}^{2}}{\sigma_{\text {R.A. }} \sigma_{\text {Decl. }}}$ & $N$ & LDC & Align & $\begin{array}{l}\text { Rate } \\
(\mathrm{Hz}) \\
\end{array}$ \\
\hline 53271.49964 & 59.1469 & 105.9933 & 9.1 & 487.1 & 146.70 & 407.2 & 267.6 & -0.99918 & 3092 & 0 & 0 & 100 \\
\hline 53285.47060 & 61.8963 & 110.1620 & 14.6 & 376.9 & 19.95 & 354.3 & 129.4 & 0.99273 & 2503 & 0 & 0 & 100 \\
\hline 53290.47919 & 62.4497 & 112.0044 & 39.6 & 2034.0 & 151.07 & 1780.2 & 984.6 & -0.99894 & 531 & 0 & 0 & 100 \\
\hline 53312.46161 & 66.0452 & 119.6805 & 5.2 & 108.2 & 158.79 & 100.9 & 39.5 & -0.98982 & 6840 & 0 & 0 & 100 \\
\hline 53334.41210 & 69.4569 & 127.2488 & 11.5 & 235.3 & 160.77 & 222.2 & 78.3 & -0.98775 & 2876 & 0 & 0 & 100 \\
\hline 53340.37952 & 70.2141 & 128.6951 & 15.0 & 165.2 & 157.64 & 152.9 & 64.4 & -0.96791 & 2056 & 0 & 0 & 100 \\
\hline 53341.34723 & 70.4709 & 129.3402 & 13.2 & 146.6 & 153.14 & 130.9 & 67.3 & -0.97539 & 3570 & 0 & 0 & 100 \\
\hline 53639.51295 & 104.4652 & 211.4488 & 16.3 & 226.6 & 149.61 & 195.6 & 115.5 & -0.98653 & 1204 & 0 & 0 & 100 \\
\hline 53663.47636 & 106.2444 & 217.1615 & 10.4 & 121.4 & 153.87 & 109.0 & 54.3 & -0.97702 & 1829 & 0 & 0 & 100 \\
\hline 53698.43902 & 110.2454 & 225.7804 & 46.8 & 894.1 & 37.76 & 707.5 & 548.8 & 0.99417 & 827 & 0 & 0 & 100 \\
\hline 53705.34654 & 108.6092 & 226.3868 & 56.3 & 1797.7 & 151.29 & 1576.9 & 864.9 & -0.99724 & 574 & 0 & 0 & 100 \\
\hline 53732.29696 & 111.8048 & 231.4902 & 20.0 & 187.4 & 156.29 & 171.8 & 77.5 & -0.95961 & 1103 & 0 & 0 & 100 \\
\hline 53753.23270 & 113.4891 & 235.7197 & 43.7 & 346.8 & 158.08 & 322.2 & 135.7 & -0.93783 & 621 & 0 & 0 & 100 \\
\hline 53789.18100 & 117.7579 & 244.2916 & 47.3 & 2294.6 & 36.86 & 1836.0 & 1377.0 & 0.99908 & 610 & 0 & 0 & 100 \\
\hline 54056.41922 & 131.6278 & 287.8511 & 35.5 & 279.5 & 159.04 & 261.3 & 105.3 & -0.93264 & 699 & 1 & 1 & 50 \\
\hline 54061.42434 & 132.3749 & 288.4474 & 30.7 & 580.4 & 161.71 & 551.1 & 184.5 & -0.98450 & 515 & 1 & 1 & 100 \\
\hline 54103.32199 & 134.6975 & 294.9159 & 44.7 & 1066.4 & 163.89 & 1024.6 & 299.0 & -0.98780 & 182 & 1 & 1 & 50 \\
\hline
\end{tabular}

Notes. All quantities are in the ICRS 2000.0 reference frame. The uncertainty values presented in these data have all been scaled by a factor of 1.73 over the formal (internal) uncertainties within each given night. Column 1 is the heliocentric modified Julian date. Columns 2 and 3 are the differential right ascension and declination between A and B, in milli-arcseconds. Columns 4 and 5 are the $1 \sigma$ uncertainties in the minor and major axes of the measurement uncertainty ellipse, in micro-arcseconds. Column $6, \phi_{\mathrm{e}}$, is the angle between the major axis of the uncertainty ellipse and the right ascension axis, measured from increasing differential right ascension through increasing differential declination (the position angle of the uncertainty ellipse's orientation is $90-\phi_{\mathrm{e}}$ ). Columns 7 and 8 are the projected uncertainties in the right ascension and declination axes, in micro-arcseconds, while Column 9 is the covariance between these. Column 10 is the number of scans taken during a given night. Column 11 is 1 if the longitudinal dispersion compensator was in use, 0 otherwise. Column 12 is 1 if the autoaligner was in use, 0 otherwise. Column 13 represents the tracking frequency of the phasereferencing camera. The quadrant was chosen such that the larger fringe contrast is designated the primary (contrast is a combination of source luminosity and interferometric visibility).

amount over the formal uncertainties. The rescaled (raw) median minor- and major-axis uncertainties are 20 (11) and 347 (200) $\mu$ as. The rescaled (raw) mean minor- and major-axis uncertainties are 27 (16) and 668 (386) $\mu$ as.

\subsection{Previous Measurements}

Previous differential astrometry measurements of $\mu$ Ori are tabulated in Table 5 of F2002. These have been included in the current fit, with identical weightings as assigned by that investigation, though it is noted that the text contains a typographical error, and the $\rho$ unit uncertainty $\sigma_{\rho}$ should be 0.024 , rather than 0.0024 mas. The time span of these measurements is much longer than that of the PHASES program and aids in solving the long-period A-B orbit, which also lifts potential fit parameter degeneracies between that orbit and those of the short-period subsystems. Measurements marked as $3 \sigma$ outliers by that investigation are omitted, resulting in 80 measurements each of separation and position angle being used for fitting. Ten new measurements have been published since that investigation and are listed in Table 2 with weights computed with the same formula as used in F2002. Two of these measurements are found to be $3 \sigma$ outliers. In total, there are 88 measurements of separation and position angle used in fitting.

F2002 also present radial velocity observations of components $\mathrm{Aa}, \mathrm{Ba}$, and $\mathrm{Bb}$. Those measurements are included in the present fit, with weightings as reported in Tables 2-4 of that paper. Measurements marked as $3 \sigma$ outliers by that investiga-
Table 2

New Non-PHASES Astrometry for $\mu$ Ori

\begin{tabular}{lcrrcc}
\hline \hline Besselian Year & $\rho$ & \multicolumn{1}{c}{$\theta$} & Weight & Outlier & Reference \\
\hline 1991.8101 & 0.330 & 31.8 & \multicolumn{1}{c}{0.1} & 1 & Fabricius et al. (2002) \\
1996.8986 & 0.306 & 13.4 & 8.7 & 0 & Horch et al. (2001) \\
1999.0153 & 0.200 & 14.8 & 10.1 & 1 & Horch et al. (2002) \\
1999.0153 & 0.196 & 13.3 & 9.7 & 1 & Horch et al. (2002) \\
1999.0153 & 0.203 & 14.9 & 10.4 & 1 & Horch et al. (2002) \\
1999.8915 & 0.150 & 11.7 & 5.8 & 1 & Horch et al. (2002) \\
1999.8915 & 0.145 & 12.5 & 5.5 & 1 & Horch et al. (2002) \\
1999.8915 & 0.154 & 11.3 & 6.1 & 1 & Horch et al. (2002) \\
2000.7653 & 0.081 & 359.4 & 2.0 & 0 & Horch et al. (2002) \\
2005.1331 & 0.179 & 26.4 & 0.2 & 1 & Scardia (2007) \\
\hline
\end{tabular}

Notes. The ten new astrometry measurements published since F2002 for $\mu$ Ori. Column 1 is the epoch of observation in years, Column 2 is the A-B separation in arcseconds, Column 3 is the position angle east of north in degrees, and Column 4 is the measurement weight on the same scale as F2002. ( $\delta$ R.A. $=\rho \sin \theta$, $\delta$ decl. $=\rho \cos \theta$.) Column 5 is 0 if the measurement is a $3 \sigma$ outlier not used in fitting, 1 otherwise. Column 6 is the original work where the measurement was published.

tion have not been included in the present analysis. In total, 442 velocities - 220 for $\mathrm{Aa}$ and 111 for each of $\mathrm{Ba}$ and $\mathrm{Bb}$-are used in fitting.

\section{ORBITAL MODELS}

The apparent motions of the centers-of-light (photocenters) of the $\mathrm{A}=\mathrm{Aa}-\mathrm{Ab}$ and $\mathrm{B}=\mathrm{Ba}-\mathrm{Bb}$ subsystems relative to each 
Table 3

Orbital Parameters for $\mu$ Ori

\begin{tabular}{|c|c|c|c|c|c|c|c|c|}
\hline \multirow{3}{*}{$\begin{array}{l}\text { Parameter } \\
\chi^{2} \\
P_{\mathrm{AB}} \text { (days) }\end{array}$} & \multicolumn{2}{|c|}{$\begin{aligned} L_{\mathrm{Aa}} & >L_{\mathrm{Ab}} \\
L_{\mathrm{Ba}} & >L_{\mathrm{Bb}}\end{aligned}$} & \multicolumn{2}{|c|}{$\begin{aligned} L_{\mathrm{Aa}} & >L_{\mathrm{Ab}} \\
L_{\mathrm{Ba}} & <L_{\mathrm{Bb}}\end{aligned}$} & \multicolumn{2}{|c|}{$\begin{aligned} L_{\mathrm{Aa}} & <L_{\mathrm{Ab}} \\
L_{\mathrm{Ba}} & >L_{\mathrm{Bb}}\end{aligned}$} & \multicolumn{2}{|c|}{$\begin{aligned} L_{\mathrm{Aa}} & <L_{\mathrm{Ab}} \\
L_{\mathrm{Ba}} & <L_{\mathrm{Bb}}\end{aligned}$} \\
\hline & \multicolumn{2}{|c|}{723.86} & \multicolumn{2}{|c|}{723.86} & \multicolumn{2}{|c|}{723.58} & \multicolumn{2}{|c|}{723.58} \\
\hline & 6813.8 & \pm 1.2 & $\ldots$ & $\ldots$ & $\ldots$ & $\ldots$ & $\ldots$ & \\
\hline$e_{\mathrm{AB}}$ & 0.7410 & \pm 0.0011 & $\ldots$ & $\ldots$ & $\ldots$ & $\ldots$ & $\ldots$ & $\ldots$ \\
\hline$i_{\mathrm{AB}}$ (degrees) & 96.028 & \pm 0.028 & $\ldots$ & $\ldots$ & $\ldots$ & $\ldots$ & $\ldots$ & $\ldots$ \\
\hline$\omega_{\mathrm{AB}}$ (degrees) & 36.712 & \pm 0.066 & $\ldots$ & $\ldots$ & $\ldots$ & $\ldots$ & $\ldots$ & $\ldots$ \\
\hline$T_{\mathrm{AB}}(\mathrm{MHJD})$ & 46090.7 & \pm 1.0 & $\ldots$ & $\ldots$ & $\ldots$ & $\ldots$ & $\ldots$ & $\ldots$ \\
\hline$\Omega_{\mathrm{AB}}$ (degrees) & 204.877 & \pm 0.011 & $\ldots$ & $\ldots$ & $\ldots$ & $\ldots$ & $\ldots$ & $\ldots$ \\
\hline$P_{\mathrm{AaAb}}($ days $)$ & 4.4475849 & $\pm 1.2 \times 10^{-6}$ & $\cdots$ & $\cdots$ & $\cdots$ & $\ldots$ & $\cdots$ & $\ldots$ \\
\hline$e_{\mathrm{AaAb}}$ & 0.0037 & \pm 0.0014 & $\ldots$ & $\ldots$ & $\ldots$ & $\ldots$ & $\ldots$ & $\ldots$ \\
\hline$i_{\mathrm{AaAb}}$ (degrees) & 47.1 & \pm 9.0 & $\ldots$ & $\ldots$ & 50.0 & \pm 8.1 & $\ldots$ & $\ldots$ \\
\hline$\omega_{\mathrm{AaAb}}$ (degrees) & 304 & \pm 21 & $\ldots$ & $\ldots$ & $\ldots$ & $\ldots$ & $\ldots$ & $\ldots$ \\
\hline$T_{\mathrm{AaAb}}(\mathrm{MHJD})$ & 43739.69 & \pm 0.26 & $\ldots$ & $\ldots$ & $\ldots$ & $\ldots$ & $\ldots$ & $\ldots$ \\
\hline$\Omega_{\mathrm{AaAb}}$ (degrees) & 50.5 & \pm 3.7 & $\ldots$ & $\ldots$ & 231.7 & \pm 3.8 & $\ldots$ & $\ldots$ \\
\hline$P_{\mathrm{BaBb}}$ (days) & 4.7835349 & $\pm 3.0 \times 10^{-6}$ & $\ldots$ & $\ldots$ & $\ldots$ & $\ldots$ & $\ldots$ & $\ldots$ \\
\hline$e_{\mathrm{BaBb}}$ & 0.0016 & \pm 0.0014 & $\ldots$ & $\ldots$ & $\ldots$ & $\ldots$ & $\ldots$ & $\ldots$ \\
\hline$i_{\mathrm{BaBb}}($ degrees $)$ & 110.71 & \pm 0.73 & $\ldots$ & $\ldots$ & $\ldots$ & $\ldots$ & $\ldots$ & $\ldots$ \\
\hline$\omega_{\mathrm{BaBb}}$ (degrees) & 217 & \pm 47 & $\ldots$ & $\ldots$ & $\ldots$ & $\ldots$ & $\ldots$ & $\ldots$ \\
\hline$T_{\mathrm{BaBb}}(\mathrm{MHJD})$ & 43746.40 & \pm 0.63 & $\ldots$ & $\ldots$ & $\ldots$ & $\ldots$ & $\ldots$ & $\ldots$ \\
\hline$\Omega_{\mathrm{BaBb}}$ (degrees) & 111.3 & \pm 3.9 & 291.3 & \pm 3.9 & 111.3 & \pm 4.0 & 291.3 & \pm 4.0 \\
\hline$M_{\mathrm{A}}\left(M_{\odot}\right)$ & 3.030 & \pm 0.069 & $\ldots$ & $\ldots$ & $\ldots$ & $\ldots$ & $\ldots$ & $\ldots$ \\
\hline$M_{\mathrm{B}}\left(M_{\odot}\right)$ & 2.746 & \pm 0.038 & $\ldots$ & $\ldots$ & $\ldots$ & $\ldots$ & $\ldots$ & $\ldots$ \\
\hline$M_{\mathrm{Ab}} / M_{\mathrm{Aa}}$ & 0.274 & \pm 0.051 & $\ldots$ & $\ldots$ & 0.259 & \pm 0.039 & $\ldots$ & $\cdots$ \\
\hline$M_{\mathrm{Bb}} / M_{\mathrm{Ba}}$ & 0.9764 & \pm 0.0022 & $\ldots$ & $\ldots$ & $\ldots$ & $\ldots$ & $\ldots$ & $\ldots$ \\
\hline$L_{\mathrm{Ab}} / L_{\mathrm{Aa}}$ & 0 & \pm 0.040 & $\ldots$ & $\ldots$ & 0.738 & \pm 0.061 & $\ldots$ & \\
\hline$L_{\mathrm{Bb}} / L_{\mathrm{Ba}}$ & 0.765 & \pm 0.055 & 1.246 & \pm 0.089 & 0.773 & \pm 0.055 & 1.233 & \pm 0.088 \\
\hline$d$ (parsecs) & 46.11 & \pm 0.28 & $\ldots$ & $\ldots$ & $\ldots$ & $\ldots$ & $\ldots$ & $\ldots$ \\
\hline$V_{0}\left(\mathrm{~km} \mathrm{~s}^{-1}\right)$ & 42.548 & \pm 0.027 & $\ldots$ & $\ldots$ & $\ldots$ & $\ldots$ & $\ldots$ & $\ldots$ \\
\hline$M_{\mathrm{Aa}}\left(M_{\odot}\right)$ & 2.38 & \pm 0.11 & $\ldots$ & $\ldots$ & 2.408 & \pm 0.092 & $\ldots$ & $\cdots$ \\
\hline$M_{\mathrm{Ab}}\left(M_{\odot}\right)$ & 0.652 & \pm 0.097 & $\ldots$ & $\ldots$ & 0.623 & \pm 0.075 & $\ldots$ & $\ldots$ \\
\hline$M_{\mathrm{Ba}}\left(M_{\odot}\right)$ & 1.389 & \pm 0.019 & $\ldots$ & $\ldots$ & $\ldots$ & $\ldots$ & $\ldots$ & $\ldots$ \\
\hline$M_{\mathrm{Bb}}\left(M_{\odot}\right)$ & 1.356 & \pm 0.019 & $\ldots$ & $\ldots$ & $\ldots$ & $\ldots$ & $\ldots$ & $\ldots$ \\
\hline$\Phi_{\mathrm{AB}-\mathrm{AaAb}}$ (degrees) & 136.7 & \pm 8.3 & $\ldots$ & $\ldots$ & 52.2 & \pm 6.1 & $\ldots$ & $\ldots$ \\
\hline$\Phi_{\mathrm{AB}-\mathrm{BaBb}}$ (degrees) & 91.2 & \pm 3.6 & 84.5 & \pm 3.6 & 91.2 & \pm 3.8 & 84.5 & \pm 3.8 \\
\hline$\Phi_{\mathrm{AaAb}-\mathrm{BaBb}}$ (degrees) & 84.6 & \pm 4.9 & 125.1 & \pm 6.0 & 126.2 & \pm 5.9 & 82.2 & \pm 4.8 \\
\hline$a_{\mathrm{AB}}$ (mas) & 273.7 & \pm 2.1 & $\ldots$ & $\ldots$ & $\ldots$ & $\ldots$ & $\ldots$ & $\ldots$ \\
\hline$a_{\mathrm{AB}}(\mathrm{AU})$ & 12.620 & \pm 0.057 & $\ldots$ & $\ldots$ & $\ldots$ & $\ldots$ & $\ldots$ & $\ldots$ \\
\hline$a_{\mathrm{AaAb}, \mathrm{COL}}(\mu \mathrm{as})$ & 358 & \pm 84 & $\ldots$ & $\ldots$ & 364 & \pm 53 & $\ldots$ & $\ldots$ \\
\hline$\%$ & 364 & \pm 53 & & & & & & \\
\hline$a_{\mathrm{AaAb}}(\mathrm{mas})$ & 1.661 & \pm 0.016 & $\cdots$ & $\ldots$ & $\ldots$ & $\ldots$ & $\ldots$ & $\ldots$ \\
\hline$a_{\mathrm{AaAb}}(\mathrm{AU})$ & 0.07659 & \pm 0.00058 & $\cdots$ & $\cdots$ & $\cdots$ & $\ldots$ & $\cdots$ & $\cdots$ \\
\hline$a_{\mathrm{BaBb}, \mathrm{COL}}(\mu \mathrm{as})$ & 102 & \pm 30 & $\ldots$ & $\ldots$ & 98 & \pm 30 & $\ldots$ & $\ldots$ \\
\hline$a_{\mathrm{BaBb}}(\mathrm{mas})$ & 1.688 & \pm 0.013 & $\ldots$ & $\ldots$ & $\ldots$ & $\ldots$ & $\ldots$ & $\ldots$ \\
\hline$a_{\mathrm{BaBb}}(\mathrm{AU})$ & 0.07780 & \pm 0.00036 & $\ldots$ & $\ldots$ & $\ldots$ & $\ldots$ & $\ldots$ & $\ldots$ \\
\hline$\pi$ (mas) & 21.69 & \pm 0.13 & $\ldots$ & $\ldots$ & $\ldots$ & $\ldots$ & $\ldots$ & $\ldots$ \\
\hline$K_{\mathrm{Aa}}$ & 1.03 & \pm 0.26 & $\ldots$ & $\ldots$ & 1.64 & \pm 0.26 & $\ldots$ & $\ldots$ \\
\hline$K_{\mathrm{Ab}}$ & & .58 & & & 1.96 & \pm 0.27 & $\ldots$ & $\ldots$ \\
\hline$K_{\mathrm{Ba}}$ & 1.72 & \pm 0.26 & 1.99 & \pm 0.26 & 1.73 & \pm 0.26 & 1.98 & \pm 0.26 \\
\hline$K_{\mathrm{Bb}}$ & 2.02 & \pm 0.26 & 1.75 & \pm 0.26 & 2.01 & \pm 0.26 & 1.75 & \pm 0.26 \\
\hline$L_{K, \mathrm{Aa}}$ & 8.3 & \pm 2.0 & $\ldots$ & $\ldots$ & 4.8 & \pm 1.2 & $\ldots$ & $\ldots$ \\
\hline$L_{K, \mathrm{Ab}}$ & 0 & \pm 0.33 & $\ldots$ & $\ldots$ & 3.52 & \pm 0.86 & $\ldots$ & $\ldots$ \\
\hline$L_{K, \mathrm{Ba}}$ & 4.4 & \pm 1.1 & 3.45 & \pm 0.84 & 4.4 & \pm 1.1 & 3.47 & \pm 0.84 \\
\hline$L_{K, \mathrm{Bb}}$ & 3.35 & \pm 0.82 & 4.3 & \pm 1.0 & 3.38 & \pm 0.82 & 4.3 & \pm 1.0 \\
\hline
\end{tabular}

Notes. Orbital parameters for $\mu$ Ori. In the second, third, and fourth solutions, ellipses indicate a parameter that changes by less than two units in the last reported digit from the previous model. In the combined fits, all parameter uncertainties have been increased by a factor of $\sqrt{\chi_{r}^{2}}=1.08$ (though $\chi_{r}^{2}$ of the combined fit is artificial due to rescaling the uncertainties of the individual data sets, this reflects the degree to which the data sets agree with each other). The first solution is strongly preferred as it produces masses and luminosities that are correlated; the second is also possible because the stars $\mathrm{Ba}$ and $\mathrm{Bb}$ are very similar. The third and fourth solutions require an unlikely luminosity for component $\mathrm{Ab}$, given its mass, and are not preferred. $a_{\mathrm{COL}}$ is the semimajor axis of the motion of the center-of-light of a subsystem, at the $K$ band. $L_{\mathrm{X}} / L_{\mathrm{Y}}$ is the $K$-band luminosity ratio between components $\mathrm{X}$ and $\mathrm{Y} . K$ is the $K$-band absolute magnitude, $L_{K}$ is the $K$-band luminosity, in solar units. For the first two solutions, the best-fit solution yields $K_{\mathrm{Ab}}$ infinite; a lower limit is determined by setting $L_{\mathrm{Ab}} / L_{\mathrm{Aa}}$ to the upper limit of its $1 \sigma$ uncertainty range. 
Table 4

New PHASES Data for $\delta$ Equ, $\kappa$ Peg, and V819 Her

\begin{tabular}{|c|c|c|c|c|c|c|c|c|c|c|c|c|c|c|}
\hline$\underline{\text { Star }}$ & $\begin{array}{c}\text { HJD- } \\
2400000.5\end{array}$ & $\begin{array}{l}\delta \text { R.A. } \\
\text { (mas) }\end{array}$ & $\begin{array}{l}\delta \text { Decl. } \\
\text { (mas) }\end{array}$ & $\begin{array}{l}\sigma_{\min } \\
(\mu \text { as })\end{array}$ & $\begin{array}{l}\sigma_{\text {maj }} \\
(\mu \text { as })\end{array}$ & $\begin{array}{c}\phi_{\mathrm{e}} \\
(\mathrm{deg})\end{array}$ & $\begin{array}{l}\sigma_{\text {R.A. }} \\
(\mu \text { as })\end{array}$ & $\begin{array}{l}\sigma_{\text {Decl. }} \\
(\mu \text { as })\end{array}$ & $\frac{\sigma_{\text {R.A..,Decl. }}^{2}}{\sigma_{\text {R.A. }}{ }^{\sigma} \text { Decl. }}$ & $N$ & LDC & Align & $\begin{array}{l}\text { Rate } \\
(\mathrm{Hz})\end{array}$ & Eclipse \\
\hline$\delta \mathrm{Equ}$ & 53508.50939 & -86.2658 & -117.6468 & 25.7 & 1872.8 & 150.49 & 1630.0 & 922.7 & -0.99949 & 263 & 0 & 0 & 100 & 1 \\
\hline$\delta$ Equ & 53550.41402 & -94.5630 & -143.7433 & 33.6 & 248.4 & 151.98 & 219.8 & 120.4 & -0.94899 & 370 & 0 & 0 & 100 & 1 \\
\hline$\delta$ Equ & 53552.38996 & -95.1309 & -144.7397 & 10.0 & 85.5 & 150.13 & 74.3 & 43.5 & -0.96437 & 1352 & 0 & 0 & 100 & 1 \\
\hline$\delta$ Equ & 53571.33634 & -99.1010 & -155.5409 & 9.8 & 68.1 & 149.71 & 59.0 & 35.4 & -0.94762 & 1089 & 0 & 0 & 100 & 1 \\
\hline$\delta$ Equ & 53584.32135 & -101.4805 & -162.5823 & 10.4 & 104.4 & 151.49 & 91.9 & 50.7 & -0.97257 & 774 & 0 & 0 & 100 & 1 \\
\hline$\delta$ Equ & 53586.30477 & -102.0695 & -163.5727 & 13.2 & 831.5 & 150.94 & 726.8 & 404.0 & -0.99930 & 639 & 0 & 0 & 100 & 1 \\
\hline$\delta$ Equ & 53607.23078 & -105.7105 & -174.9135 & 3.1 & 80.6 & 148.50 & 68.8 & 42.2 & -0.99617 & 4856 & 0 & 0 & 100 & 1 \\
\hline$\delta$ Equ & 53613.22582 & -107.1684 & -177.7125 & 6.7 & 243.8 & 150.28 & 211.8 & 121.0 & -0.99794 & 1601 & 0 & 0 & 100 & 1 \\
\hline$\delta$ Equ & 53614.22329 & -106.8636 & -178.5240 & 5.9 & 174.7 & 150.30 & 151.8 & 86.7 & -0.99697 & 1597 & 0 & 0 & 100 & 1 \\
\hline$\delta$ Equ & 53637.20185 & -111.2007 & -189.7814 & 12.3 & 357.4 & 157.38 & 330.0 & 137.9 & -0.99531 & 570 & 0 & 0 & 100 & 1 \\
\hline$\delta$ Equ & 53656.13045 & -114.1207 & -198.7179 & 16.3 & 215.1 & 154.15 & 193.8 & 94.9 & -0.98163 & 526 & 0 & 0 & 100 & 1 \\
\hline$\delta$ Equ & 53874.48665 & -133.8986 & -275.5257 & 12.3 & 424.2 & 147.53 & 357.9 & 228.0 & -0.99794 & 264 & 0 & 0 & 100 & 1 \\
\hline$\delta$ Equ & 53909.42854 & -134.0082 & -283.8970 & 6.1 & 58.2 & 152.62 & 51.7 & 27.3 & -0.96785 & 2646 & 0 & 1 & 50 & 1 \\
\hline$\delta$ Equ & 53957.31149 & -133.6026 & -293.0248 & 9.0 & 405.5 & 154.61 & 366.4 & 174.1 & -0.99836 & 1127 & 1 & 1 & 50 & 1 \\
\hline$\delta$ Equ & 53970.25612 & -133.8368 & -294.8599 & 5.7 & 50.6 & 153.56 & 45.3 & 23.1 & -0.96133 & 2387 & 1 & 1 & 50 & 1 \\
\hline$\delta$ Equ & 53971.25621 & -133.8811 & -294.9923 & 7.3 & 62.3 & 152.11 & 55.1 & 29.8 & -0.96095 & 1682 & 1 & 1 & 50 & 1 \\
\hline$\delta$ Equ & 53977.26493 & -133.7524 & -295.9196 & 5.3 & 39.3 & 157.55 & 36.4 & 15.8 & -0.93151 & 3320 & 1 & 1 & 50 & 1 \\
\hline$\delta$ Equ & 54003.21361 & -133.0033 & -299.3443 & 16.7 & 206.4 & 161.12 & 195.4 & 68.6 & -0.96621 & 835 & 1 & 1 & 50 & 1 \\
\hline$\delta$ Equ & 54005.19180 & -133.0818 & -299.4815 & 11.6 & 115.3 & 157.21 & 106.4 & 45.9 & -0.96173 & 1614 & 1 & 1 & 50 & 1 \\
\hline$\delta$ Equ & 54028.10922 & -132.3883 & -301.8627 & 13.3 & 406.9 & 153.38 & 363.9 & 182.7 & -0.99668 & 632 & 1 & 1 & 50 & 1 \\
\hline$\delta$ Equ & 54037.11296 & -132.9388 & -302.3034 & 11.0 & 319.6 & 159.14 & 298.7 & 114.3 & -0.99468 & 556 & 1 & 1 & 100 & 1 \\
\hline$\delta$ Equ & 54230.50355 & -116.0819 & -301.5397 & 13.1 & 508.2 & 146.70 & 424.8 & 279.2 & -0.99843 & 933 & 1 & 1 & 50 & 1 \\
\hline$\delta$ Equ & 54266.47236 & -111.8997 & -297.2144 & 13.2 & 112.0 & 158.12 & 104.1 & 43.5 & -0.94550 & 1315 & 1 & 1 & 50 & 1 \\
\hline$\kappa \mathrm{Peg}$ & 53494.50786 & 104.6067 & 43.3687 & 13.6 & 645.9 & 143.23 & 517.5 & 386.8 & -0.99904 & 702 & 0 & 0 & 100 & 1 \\
\hline$\kappa$ Peg & 53586.36471 & 83.9658 & 55.9066 & 2.2 & 11.5 & 166.51 & 11.2 & 3.4 & -0.75093 & 5532 & 0 & 0 & 100 & 1 \\
\hline$\kappa \mathrm{Peg}$ & 53637.29586 & 71.2323 & 62.9289 & 6.3 & 53.5 & 173.82 & 53.2 & 8.5 & -0.66476 & 1639 & 0 & 0 & 100 & 1 \\
\hline$\kappa \mathrm{Peg}$ & 53921.45172 & 25.0960 & 85.9815 & 43.1 & 10007.6 & 160.71 & 9445.7 & 3306.4 & -0.99990 & 615 & 0 & 1 & 50 & 1 \\
\hline$\kappa$ Peg & 53963.35858 & -9.1846 & 98.3661 & 6.2 & 79.3 & 165.54 & 76.8 & 20.7 & -0.95159 & 2244 & 1 & 1 & 100 & 1 \\
\hline$\kappa$ Peg & 53978.32708 & -14.0804 & 99.4673 & 2.7 & 17.5 & 162.83 & 16.8 & 5.8 & -0.87056 & 5863 & 1 & 1 & 50 & 1 \\
\hline$\kappa \mathrm{Peg}$ & 53995.24384 & -17.0621 & 101.1081 & 11.5 & 514.2 & 159.38 & 481.3 & 181.5 & -0.99770 & 389 & 1 & 1 & 100 & 1 \\
\hline$\kappa$ Peg & 54003.32618 & -22.4829 & 101.1592 & 10.9 & 849.5 & 3.09 & 848.2 & 47.1 & 0.97271 & 1590 & 1 & 1 & 50 & 1 \\
\hline$\kappa$ Peg & 54008.31093 & -21.6029 & 102.0991 & 4.6 & 114.1 & 2.48 & 113.9 & 6.7 & 0.73308 & 2850 & 1 & 1 & 50 & 1 \\
\hline$\kappa$ Peg & 54075.12667 & -38.5846 & 107.2133 & 7.0 & 183.8 & 3.06 & 183.5 & 12.0 & 0.81528 & 1756 & 1 & 1 & 50 & 1 \\
\hline$\kappa \mathrm{Peg}$ & 54265.39966 & -84.8163 & 119.9685 & 8.2 & 389.0 & 142.94 & 310.4 & 234.5 & -0.99904 & 3661 & 1 & 1 & 50 & 1 \\
\hline V819 Her & 53109.47951 & 49.6406 & -84.4966 & 7.3 & 282.5 & 158.77 & 263.4 & 102.5 & -0.99707 & 2011 & 0 & 0 & 100 & 1 \\
\hline V819 Her & 53110.48183 & 48.0946 & -84.1334 & 11.9 & 600.4 & 159.53 & 562.5 & 210.3 & -0.99819 & 1334 & 0 & 0 & 100 & 1 \\
\hline V819 Her & 53123.45772 & 49.1860 & -85.9318 & 18.1 & 507.8 & 162.47 & 484.3 & 153.9 & -0.99240 & 1378 & 0 & 0 & 100 & 0 \\
\hline V819 Her & 53130.44208 & 48.4778 & -86.4135 & 6.6 & 205.8 & 162.94 & 196.7 & 60.7 & -0.99360 & 2537 & 0 & 0 & 100 & 1 \\
\hline V819 Her & 53137.43044 & 48.3928 & -87.1396 & 14.0 & 280.2 & 164.34 & 269.9 & 76.8 & -0.98202 & 1226 & 0 & 0 & 100 & 1 \\
\hline V819 Her & 53144.42426 & 47.7017 & -87.6612 & 25.1 & 1039.6 & 167.13 & 1013.5 & 232.9 & -0.99386 & 897 & 0 & 0 & 100 & 1 \\
\hline V819 Her & 53145.39541 & 48.3082 & -87.8013 & 13.7 & 316.5 & 161.59 & 300.3 & 100.8 & -0.98964 & 1673 & 0 & 0 & 100 & 1 \\
\hline V819 Her & 53168.33949 & 47.0275 & -89.7513 & 15.0 & 339.9 & 162.93 & 325.0 & 100.8 & -0.98778 & 1409 & 0 & 0 & 100 & 1 \\
\hline V819 Her & 53172.35221 & 47.3441 & -90.1337 & 6.3 & 170.0 & 168.29 & 166.5 & 35.1 & -0.98309 & 2560 & 0 & 0 & 100 & 1 \\
\hline V819 Her & 53173.33202 & 47.1604 & -90.3599 & 8.0 & 77.4 & 33.97 & 64.4 & 43.8 & 0.97548 & 2904 & 0 & 0 & 100 & 1 \\
\hline V819 Her & 53181.33391 & 46.4333 & -90.7857 & 7.5 & 174.6 & 169.71 & 171.8 & 32.1 & -0.97114 & 2795 & 0 & 0 & 100 & 0 \\
\hline V819 Her & 53182.33164 & 46.5646 & -91.0136 & 13.9 & 333.2 & 169.62 & 327.8 & 61.6 & -0.97328 & 2014 & 0 & 0 & 100 & 1 \\
\hline V819 Her & 53186.30448 & 45.6584 & -91.1213 & 18.2 & 426.3 & 166.80 & 415.1 & 99.0 & -0.98197 & 706 & 0 & 0 & 100 & 1 \\
\hline V819 Her & 53187.30462 & 46.1427 & -91.2225 & 13.0 & 441.5 & 166.94 & 430.1 & 100.6 & -0.99110 & 1578 & 0 & 0 & 100 & 1 \\
\hline V819 Her & 53197.26851 & 46.1852 & -92.1522 & 4.8 & 117.1 & 164.87 & 113.0 & 30.9 & -0.98715 & 5218 & 0 & 0 & 100 & 0 \\
\hline V819 Her & 53198.24258 & 46.2937 & -92.2555 & 5.7 & 54.6 & 160.37 & 51.5 & 19.1 & -0.94836 & 5404 & 0 & 0 & 100 & 0 \\
\hline V819 Her & 53199.29186 & 44.0252 & -91.9446 & 24.7 & 1645.4 & 171.42 & 1627.0 & 246.7 & -0.99488 & 946 & 0 & 0 & 100 & 0 \\
\hline V819 Her & 53208.25236 & 46.4303 & -92.4901 & 6.6 & 181.6 & 37.67 & 143.8 & 111.1 & 0.99718 & 6558 & 0 & 0 & 100 & 0 \\
\hline V819 Her & 53214.24077 & 45.6337 & -93.3429 & 5.5 & 125.9 & 169.45 & 123.8 & 23.7 & -0.97194 & 5251 & 0 & 0 & 100 & 1 \\
\hline V819 Her & 53215.23094 & 45.6364 & -93.5172 & 4.8 & 110.6 & 167.53 & 108.0 & 24.3 & -0.97962 & 5723 & 0 & 0 & 100 & 1 \\
\hline V819 Her & 53221.22209 & 46.2559 & -92.9726 & 8.8 & 342.2 & 38.91 & 266.3 & 215.0 & 0.99860 & 3998 & 0 & 0 & 100 & 1 \\
\hline V819 Her & 53228.20946 & 45.0884 & -94.3310 & 7.2 & 100.6 & 169.45 & 98.9 & 19.7 & -0.92813 & 3180 & 0 & 0 & 100 & 0 \\
\hline V819 Her & 53229.22073 & 45.2196 & -94.5160 & 6.4 & 80.0 & 172.84 & 79.4 & 11.8 & -0.83914 & 3905 & 0 & 0 & 100 & 1 \\
\hline V819 Her & 53233.18295 & 45.0993 & -94.8458 & 6.0 & 64.7 & 167.67 & 63.2 & 15.0 & -0.91188 & 3303 & 0 & 0 & 100 & 1 \\
\hline V819 Her & 53234.20151 & 44.8269 & -94.7637 & 7.6 & 37.8 & 172.74 & 37.5 & 8.9 & -0.51352 & 3701 & 0 & 0 & 100 & 1 \\
\hline V819 Her & 53235.21764 & 45.2153 & -94.9183 & 8.5 & 107.1 & 176.57 & 106.9 & 10.6 & -0.60015 & 2094 & 0 & 0 & 100 & 1 \\
\hline V819 Her & 53236.16733 & 44.4598 & -94.8862 & 4.7 & 78.1 & 166.59 & 76.0 & 18.7 & -0.96552 & 6684 & 0 & 0 & 100 & 0 \\
\hline V819 Her & 53249.16006 & 44.2467 & -95.8032 & 4.3 & 87.9 & 172.71 & 87.2 & 12.0 & -0.93121 & 5428 & 0 & 0 & 100 & 1 \\
\hline V819 Her & 53466.52265 & 31.4132 & -102.8881 & 9.9 & 204.3 & 163.01 & 195.4 & 60.4 & -0.98524 & 3031 & 0 & 0 & 100 & 1 \\
\hline V819 Her & 53481.50628 & 30.4432 & -103.3348 & 11.1 & 311.1 & 38.18 & 244.6 & 192.5 & 0.99731 & 3301 & 0 & 0 & 100 & 0 \\
\hline V819 Her & 53494.45305 & 29.5257 & -103.1671 & 18.6 & 251.9 & 163.98 & 242.2 & 71.8 & -0.96316 & 1355 & 0 & 0 & 100 & 1 \\
\hline V819 Her & 53585.24930 & 23.9818 & -102.6116 & 10.1 & 114.7 & 174.02 & 114.0 & 15.6 & -0.76046 & 1479 & 0 & 0 & 100 & 1 \\
\hline V819 Her & 53874.42827 & 1.2427 & -88.7776 & 6.8 & 99.5 & 168.07 & 97.4 & 21.6 & -0.94665 & 2358 & 0 & 0 & 100 & 1 \\
\hline V819 Her & 53956.21398 & -5.0810 & -81.9129 & 9.2 & 341.7 & 170.23 & 336.8 & 58.7 & -0.98729 & 4028 & 1 & 1 & 50 & 0 \\
\hline
\end{tabular}

Notes. All quantities are in the ICRS 2000.0 reference frame. The uncertainty values presented in these data have not been rescaled. Column 1 is the star name. Columns $2-14$ are as Columns 1-13 in Table 1. Column 15 is 0 if the measurement was taken during a subsystem eclipse, 1 otherwise (V819 Her only). 
other are given by the model

$$
\begin{aligned}
\overrightarrow{y_{\mathrm{obs}}}= & \overrightarrow{r_{\mathrm{A}-\mathrm{B}}}+\frac{M_{\mathrm{Ab}} / M_{\mathrm{Aa}}-L_{\mathrm{Ab}} / L_{\mathrm{Aa}}}{\left(1+M_{\mathrm{Ab}} / M_{\mathrm{Aa}}\right)\left(1+L_{\mathrm{Ab}} / L_{\mathrm{Aa}}\right)} \overrightarrow{r_{\mathrm{Aa}-\mathrm{Ab}}} \\
& -\frac{M_{\mathrm{Bb}} / M_{\mathrm{Ba}}-L_{\mathrm{Bb}} / L_{\mathrm{Ba}}}{\left(1+M_{\mathrm{Bb}} / M_{\mathrm{Ba}}\right)\left(1+L_{\mathrm{Bb}} / L_{\mathrm{Ba}}\right)} \overrightarrow{\mathrm{Ba}-\mathrm{Bb}}
\end{aligned}
$$

corresponding to a four-body hierarchical dynamical system (HDS). The quantities $M$ are component masses and $L$ are component luminosities, and each of the summed vectors is determined by a two-body Keplerian model. This model is used to fit the astrometric data; note that the total masses from the $\mathrm{Aa}-\mathrm{Ab}$ and $\mathrm{Ba}-\mathrm{Bb}$ orbits also show up as component masses for the $\mathrm{A}-\mathrm{B}$ orbit, linking them, and that the mass ratios and luminosity ratios appear as additional parameters, degenerate with each other. The radial velocities are fitted by a simple superposition of individual Keplerians; these determine mass ratios, and the luminosity ratios become nondegenerate parameters. The luminosity ratios are primarily constrained by the $K$-band PHASES data; the other astrometric data are not precise enough to detect the subsystem motions.

The combined simultaneous fit to all data sets has 26 free parameters and 626 degrees of freedom. The parameters used are listed in the top half of Table 3 with their associated best-fit values and $1 \sigma$ uncertainties. It should be noted that while the $\mathrm{Ba}-\mathrm{Bb}$ eccentricity has been allowed to vary as a free parameter, the best-fit value is consistent with zero and could have been fixed; the other parameters are not changed significantly by doing so. Quantities of interest derived from those parameters are listed in the second half of that table, with corresponding uncertainties derived from first-order uncertainty propagation. The apparent center-of-light wobbles of the $\mathrm{Aa}-\mathrm{Ab}$ and $\mathrm{Ba}-\mathrm{Bb}$ subsystems are plotted in Figure 1; the A-B and RV orbits were plotted in F2002 and are not significantly different in the present model.

Including PHASES measurements in the fit introduces the ability to evaluate the inclination and luminosity ratio of the $\mathrm{Aa}-\mathrm{Ab}$ system and angles of the nodes of the $\mathrm{Aa}-\mathrm{Ab}$ and $\mathrm{Ba}-$ $\mathrm{Bb}$ systems, quantities that were entirely unconstrained in the F2002 study. The A-B angular parameters have much smaller uncertainties than in F2002 (the $\Omega_{\mathrm{AB}}, i_{\mathrm{AB}}$, and $\omega_{\mathrm{AB}}$ uncertainties are reduced by $13 \times, 6 \times$, and $5 \times$, respectively). Uncertainties in the A-B period, eccentricity, and epoch of periastron passage are improved by a factor of 2 or more. Most other fit parameters are constrained only marginally better than in F2002.

\subsection{Relative Orbital Inclinations}

In systems with three or more stars, studying the system coplanarity is of interest for understanding the formation and evolution of multiples (Sterzik \& Tokovinin 2002). To determine this without ambiguity, one must have both visual and RV orbital solutions for pairs of interest. Previously, this was available only in six triples (for a listing, see Muterspaugh et al. 2006a). The reason mutual inclination measurements have been rare is due to the observational challenges these systems present: RV signals are largest for compact pairs of stars, whereas astrometry prefers wider pairs. The "wide" pair must be studied with RV and thus have an orbital period (and corresponding separation) as short as the two-component binaries that are already challenging to visual studies. The "narrow" pair is even smaller. The mutual inclination between two orbits is given by

$$
\cos \Phi=\cos i_{1} \cos i_{2}+\sin i_{1} \sin i_{2} \cos \left(\Omega_{1}-\Omega_{2}\right)
$$

where $i_{1}$ and $i_{2}$ are the orbital inclinations, and $\Omega_{1}$ and $\Omega_{2}$ are the longitudes of the ascending nodes. If only velocities are available for one system, the orientation of that orbit is unknown (even if it is eclipsing, the longitude of the ascending node is unknown). If velocities are unavailable for a given orbit, there is a degeneracy in which node is ascending - two values separated by 180 degrees are possible. This gives two degenerate solutions for the mutual inclination (not necessarily separated by 180 degrees).

Even when a center-of-light astrometric orbit is available for the narrow pair, there can be a degeneracy between the node which is ascending and the luminosity ratio. Having found one possible luminosity ratio $L_{\mathrm{Ab}} / L_{\mathrm{Aa}}=L_{1}$, it can be shown that the other possible solution, corresponding to changing the ascending node by 180 degrees, is given by

$$
L_{2}=\frac{2 R+R L_{1}-L_{1}}{1+2 L_{1}-R}
$$

where $R$ is the mass ratio $M_{\mathrm{Ab}} / M_{\mathrm{Aa}}$. In previous studies, support data have been available to lift that degeneracy. For example, in the V819 Her system (Muterspaugh et al. 2006a), the two possible luminosity ratios were 0.26 and 1.89 . The eclipsing nature of the $\mathrm{Ba}-\mathrm{Bb}$ pair lifted that degeneracy because it helped establish that the luminosity ratio is much less than 1 . Similarly, in the $\kappa$ Peg system (Muterspaugh et al. 2006b), the spectra used for RV also show that component $\mathrm{Bb}$ is much fainter than $\mathrm{Ba}$, again lifting the degeneracy (a luminosity ratio either nearly zero or 1.9 both possible).

For a quadruple system, there are as many as four degenerate fit solutions. For $\mu$ Ori, a global minimum $\chi^{2}$ is found with longitude of the ascending node $\Omega_{\mathrm{AaAb}}=231.7 \pm 3.8$ degrees and $L_{\mathrm{Ab}} / L_{\mathrm{Aa}}=0.738 \pm 0.061$. The alternative pair of these parameters solving Equation (3) would force the luminosity ratio to a negative value (but close to zero within uncertainties). However, searching for solutions with nonnegative luminosity ratios near zero yields a fit solution with only slightly larger $\chi^{2}$, and the node at roughly 180 degrees difference $\left(\Omega_{\mathrm{AaAb}}=\right.$ $50.5 \pm 3.7$ and $L_{\mathrm{Ab}} / L_{\mathrm{Aa}}=0 \pm 0.040$ ). All parameters other than the node angle and luminosity ratio vary between the two models by amounts less than the fit uncertainties. The two solutions find $M_{\mathrm{Ab}} / M_{\mathrm{Aa}}=0.259 \pm 0.039$ or $0.274 \pm 0.051$, respectively; given the rough scaling $L \propto M^{4}$ (Smith 1983), it is very likely that the larger luminosity ratio is incorrect. The larger luminosity ratio would also imply that $\mathrm{Ab}$ is as bright as $\mathrm{Ba}$ or $\mathrm{Bb}$. However, component $\mathrm{Ab}$ is not observed in the spectrum while $\mathrm{Ba}$ and $\mathrm{Bb}$ are. This suggests that $\mathrm{Ab}$ is faint, though it is also possible to explain this lack of $\mathrm{Ab}$ lines by postulating that it is rapidly rotating. However, given that the other stars are not rapid rotators, there is little evidence to support $\mathrm{Ab}$ as a rapid rotator. It is concluded that the luminosity ratio near zero is strongly preferred, despite the slightly worse $\chi^{2}$ fit. Both fits are reported in Table 3, but the rest of the discussion in this paper refers only to the preferred solution for $\mathrm{Aa}-\mathrm{Ab}$. This degeneracy can be fully lifted by a single epoch image with a closure phase capable interferometer with sufficient angular resolution (such as the Navy Prototype Optical Interferometer; Armstrong et al. 1998).

For each of the Aa-Ab solutions, there exist two solutions for the $\mathrm{Ba}-\mathrm{Bb}$ pair. In these cases, no negative luminosity ratios are found; the degeneracy is perfect and $\chi^{2}$ of the fits are identical. $\Omega_{\mathrm{BaBb}}$ differs by 180 degrees in the two orbits, and the luminosity ratio $L_{\mathrm{Bb}} / L_{\mathrm{Ba}}$ switches between being larger (at $\Omega_{\mathrm{BaBb}}=291$ degrees) or smaller 

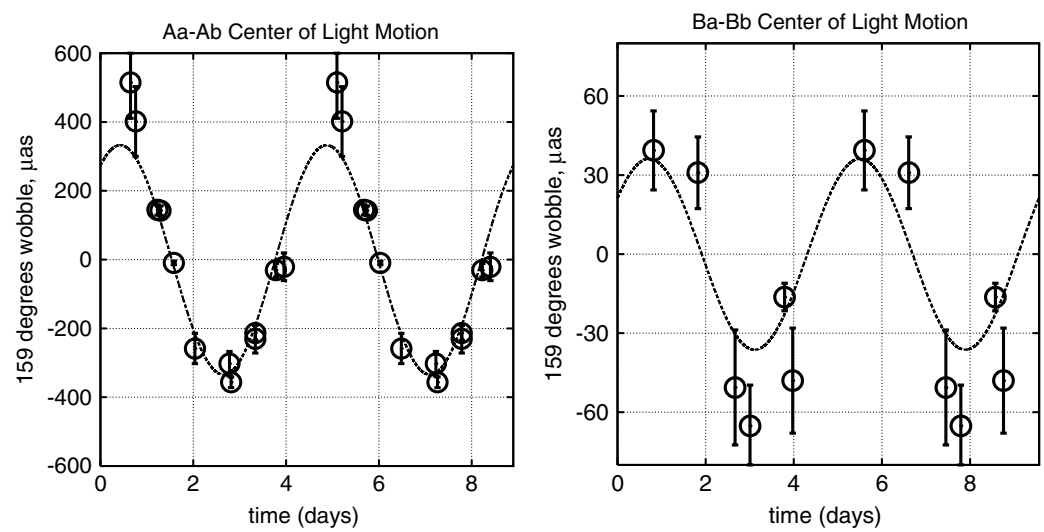

Figure 1. Astrometric orbits of $\mu$ Ori $\mathrm{Aa}-\mathrm{Ab}$ and $\mathrm{Ba}-\mathrm{Bb}$, phase-wrapped about their respective orbital periods. Phase zero is at the epoch of periastron passage ( $T$ ), and each plot is repeated for two cycles (and each measurement is plotted twice) to allow for continuity at all parts of the graph. In both cases, the motions of the A-B system and the other subsystem have been removed. The projection axis shown for each is 159 degrees east of north (equivalent to position angle 291 degrees), well aligned with the minor axis of many PHASES observations. The plotted uncertainties are those projected along this axis, and have been increased by a factor of 1.73 over the formal uncertainties, to reflect excess noise within the PHASES set. On the left is the motion of Aa-Ab; for clarity, only those observations with rescaled and projected uncertainties less than $200 \mu$ as have been plotted; on the right is the motion of $\mathrm{Ba}-\mathrm{Bb}$, for which the cutoff was at $30 \mu$ as.

(at 111 degrees) than unity; all other parameters remain unchanged. Both solutions are near unity, and the stars have very similar masses $\left(M_{\mathrm{Bb}} / M_{\mathrm{Ba}}=0.9764 \pm 0.0022\right)$. While the solution for which $\mathrm{Bb}$ is less luminous than $\mathrm{Ba}$ is slightly more consistent because it correlates to the mass ratio, it is conceivable that the other solution is correct. Thus, the solution for which $\mathrm{Bb}$ is less luminous than $\mathrm{Ba}$ is slightly preferred, but not as conclusively as for the $\mathrm{Aa}-\mathrm{Ab}$ case, where the differences between the stars are more significant. Thus, both possibilities are considered in the remainder of this paper.

\subsection{Evidence for Kozai Cycles with Tidal Friction?}

Of particular interest is the potential for Kozai oscillations between orbital inclination and eccentricity in the narrow pairs (Kozai 1962), which can affect the orbital evolution of the system. These occur independently of distances or component masses, with the only requirement being that the mutual inclination be between 39.2 and $180-39.2=140.8$ degrees. Other effects that cause precession can increase the value of this critical angle.

Fabrycky \& Tremaine (2007) predict a buildup of mutual inclinations near 40 and 140 degrees by the combined effects of Kozai Cycles with Tidal Friction (KCTF) for triples whose short-period subsystems have periods between 3 and 10 days. The mutual inclination of $\mu$ Ori $\mathrm{AB}-\mathrm{AaAb}$ is near 140 degrees, leading one to consider if this trend is starting to be seen. The systems with unambiguous mutual inclinations break down as follows:

- Five systems are outside of the 3-10 day inner period range. These systems do not meet the criteria to be included in testing the buildup prediction:

(1) V819 Her $(\Phi=26.3 \pm 1.5$ degrees, $2.23 \mathrm{~d}$; see Section 4),

(2) Algol $(\Phi=98.8 \pm 4.9$ degrees, $2.9 \mathrm{~d}$; Lestrade et al. 1993; Pan et al. 1993),

(3) $\eta \operatorname{Vir}(\Phi=30.8 \pm 1.3$ degrees, $72 \mathrm{~d}$; Hummel et al. 2003),

(4) $\xi$ Uma $\mathrm{ABC}(\Phi=132.1$ degrees, $670 \mathrm{~d}$; Heintz 1996),

(5) $\epsilon$ Hya $\operatorname{ABC}(\Phi=39.4$ degrees, 5500 d; Heintz 1996).

These fall outside the 3-10 day range of inner-binary periods applicable to the prediction in Fabrycky \& Tremaine (2007). However, it is worth noting that the mutual inclinations of $\xi$
UMa and $\epsilon$ Hya are near 140 and 40 degrees, respectively, and in V819 Her and $\eta$ Vir the values are outside the 40-140 degrees range, so neither would have been predicted to undergo Kozai cycles or KCTF. Algol has a nearly perpendicular alignment, though the dynamics of Algol are different due to quadrupole distortions in the semidetached stars; Algol's alignment has been explained by Eggleton \& Kiseleva-Eggleton (2001).

- No systems are outside the 40-140 degrees range, while also in the 3-10 day inner period range.

- Two systems are between 40 and 140 degrees and in the 3-10 day inner period range. These systems would not support the KCTF-driven buildup near 40 and 140 degrees:

(1) $\mu$ Ori $\mathrm{AB}-\mathrm{BaBb}(\Phi=91.2 \pm 3.6$ or $84.5 \pm 3.6$ degrees are possible, $4.78 \mathrm{~d})$

(2) $88 \mathrm{Tau} \mathrm{AaAb}-\mathrm{Ab} 1 \mathrm{Ab} 2(\Phi=82.0 \pm 3.3$ or $58 \pm 3.3$ degrees are possible, $7.89 \mathrm{~d}$; Lane et al. 2007).

While mutual inclination degeneracies continue to exist in both systems, all possible values are in this range.

- Three systems are near 40 or 140 degrees, and in the 3-10 day inner period range. These systems would appear to support the KCTF prediction:

(1) $\mu$ Ori $\mathrm{AB}-\mathrm{AaAb}(\Phi=136.7 \pm 8.3$ degrees, $4.45 \mathrm{~d})$,

(2) $88 \mathrm{Tau} \mathrm{AaAb}-\mathrm{Aa} 1 \mathrm{Aa} 2(\Phi=143.3 \pm 2.5$ degrees, $3.57 \mathrm{~d}$; Lane et al. 2007),

(3) $\kappa \operatorname{Peg}(\Phi=43.4 \pm 3.9$ degrees, $5.97 \mathrm{~d}$; see Section 4).

In total, three of the five systems meeting the criteria for testing the KCTF prediction do appear near the peak points of 40 and 140 degrees. Following Equations (1), (22), and (35) in Fabrycky \& Tremaine (2007), in the presence of general relativity (GR) precession one expects the critical angles for $\mu$ Ori $\mathrm{AB}-\mathrm{AaAb}$ and $\kappa$ Peg to be increased from 39.2 degrees to $\sim 68\left(\tau \dot{\omega}_{\mathrm{GR}}=2.3\right)$ and $\sim 54$ degrees $\left(\tau \dot{\omega}_{\mathrm{GR}}=1.3\right)$, respectively, while for 88 Tau AaAb-Aa1Aa2 GR precession dominates no matter the inclination $\left(\tau \dot{\omega}_{\mathrm{GR}}=17\right)$. Thus, Kozai oscillations are suppressed by precession in these systems' current states. However, it is possible these were present at earlier stages in the systems' histories and their current configurations were still reached through $\mathrm{KCTF}$ - the inner binaries 
may have originally been more widely separated, in which case GR effects would have been reduced.

Alternatively, $\mu$ Ori AB-BaBb and 88 Tau AaAb-Ab1Ab2 both lie well within the range of predicted Kozai cycles, even including GR precession (which raises the critical angles to $\sim 58$ $\left(\tau \dot{\omega}_{\mathrm{GR}}=1.6\right)$ and $\sim 50$ degrees $\left(\tau \dot{\omega}_{\mathrm{GR}}=0.9\right)$, respectively $)$.

Several more systems with double visual orbits but lacking $\mathrm{RV}$ for at least one subsystem component are listed by Sterzik \& Tokovinin (2002). Two more (HD 150680 and HD 214608) are mentioned by Orlov \& Petrova (2000) and one more (HD 108500) by Orlov \& Zhuchkov (2005). Of these, the inner pair in HD 150680 is doubtful and listing HD 214608 as having a double visual orbit appears to be in error. In the paper cited by Orlov \& Petrova (2000) for HD 214608, Duquennoy (1987) reveals it to be a double spectroscopic system, but points out that the visual elements of the inner pair are unconstrained. The value of 150 degrees for the node seems to have been taken as nominal from the outer system (whose true ascending node is 180 degrees different). Because the nodes cannot be distinguished in the visual-only pairs, two values of the mutual inclinations are equally possible for each. Furthermore, all have inner systems with periods longer than 300 days. Thus, these cannot provide further direction on testing the KCTF prediction.

\subsection{Masses and Distance}

Components $\mathrm{Ba}$ and $\mathrm{Bb}$ are each determined to $1.4 \%$, Aa to $5 \%$, while the lowest mass member, $\mathrm{Ab}$, is uncertain at the $15 \%$ level. The individual masses of $\mathrm{Aa}$ and $\mathrm{Ab}$ have not been previously determined; this study enables the exploration of the natures of those stars. Both are members of star classes of interest: $\mathrm{Aa}$ is of spectral type $\mathrm{Am}$, and $\mathrm{Ab}$ has a mass in the range of late-K dwarfs. Through their physical association, it can be assumed that both are co-evolved with $\mathrm{Ba}$ and $\mathrm{Bb}$, each within the mass range for which stellar models have been well calibrated through observation.

The masses of $\mathrm{Ba}$ and $\mathrm{Bb}$ are determined only slightly better (less than a factor of 2 improvement) over the previous study by F2002. Similarly, the distance is determined to $0.6 \%$, a slight improvement. The Hipparcos-based (Perryman et al. 1997) parallax values discussed by Søderhjelm (1999) (21.5 \pm 0.8 mas in the original evaluation, revised to $20.8 \pm 0.9$ mas when the binary nature was considered) are consistent with, but less well constrained than, the current value of $21.69 \pm 0.13$ mas.

\subsection{Component Luminosities}

The 2MASS $K$-band magnitude for $\mu$ Ori is $m_{\text {Total }}=$ $3.637 \pm 0.260$ (Skrutskie et al. 2006). F2002 gives the difference between the luminosities of A and B in several bands. Unfortunately, none of these was taken near the $K$ band $(2.2 \mu \mathrm{m})$ where PTI operates. However, a Keck adaptive optics image of $\mu$ Ori was obtained on MJD 53227 with a narrow band $\mathrm{H}_{2} 2-1$ filter centered at 2.2622 microns. The $\mathrm{A}-\mathrm{B}$ differential magnitude in this band is $m_{\mathrm{A}}-m_{\mathrm{B}}=\Delta m_{\mathrm{AB}}=-0.073 \pm 0.007$ magnitudes; this measurement is reported for the first time here.

The combined orbital fit provides the system distance $d=$ $46.11 \pm 0.28$ parsecs and the luminosity ratios. The combined set of $m_{\mathrm{Total}}, \Delta m_{\mathrm{AB}}, d, L_{\mathrm{Ab}} / L_{\mathrm{Aa}}$, and $L_{\mathrm{Bb}} / L_{\mathrm{Ba}}$ determines the component luminosities. Using first-order error propagation, the $K$-band luminosities are $L_{K, \mathrm{Aa}}=8.3 \pm 2.0$ solar and less than a third solar for $\mathrm{Ab}$. Components $\mathrm{Ba}$ and $\mathrm{Bb}$ have $K$ band luminosities of either $L_{K, \mathrm{Ba}}=4.4 \pm 1.1$ and $L_{K, \mathrm{Bb}}=$ $3.35 \pm 0.82$, or $L_{K, \mathrm{Ba}}=3.45 \pm 0.84$ and $L_{K, \mathrm{Bb}}=4.3 \pm 1.0$

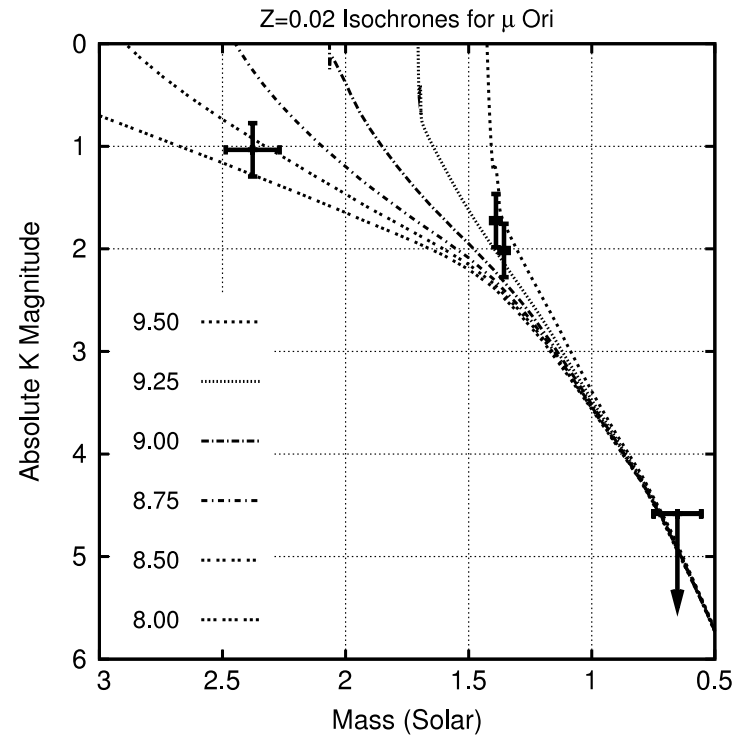

Figure 2. Stellar evolution models predicting isochrones for the elements of the $\mu$ Ori system show disagreement between components Aa with $\mathrm{Ba}$ and $\mathrm{Bb}$. Curve labels give values of $\log$ (age/years). Component Aa provides the most leverage for determining the system age, though its evolution may have been altered by tidal friction.

Table 5

Orbital Parameters for $\delta$ Equ

\begin{tabular}{lcl}
\hline \hline Parameter & Value & Uncertainty \\
\hline$P$ (days) & 2084.03 & \pm 0.10 \\
$T$ (MHJD) & 53112.071 & \pm 0.052 \\
$e$ & 0.436851 & \pm 0.000025 \\
$a$ (mas) & 231.9650 & \pm 0.0080 \\
$V_{0, \text { Lick }}\left(\mathrm{km} \mathrm{s}^{-1}\right)$ & -15.40 & \pm 0.11 \\
$V_{0, \mathrm{DAO}}\left(\mathrm{km} \mathrm{s}^{-1}\right)$ & -15.875 & \pm 0.080 \\
$V_{0, \mathrm{C}}\left(\mathrm{km} \mathrm{s}^{-1}\right)$ & -15.73 & \pm 0.10 \\
$M_{1}\left(M_{\odot}\right)$ & 1.192 & \pm 0.012 \\
$M_{2}\left(M_{\odot}\right)$ & 1.187 & \pm 0.012 \\
$M_{1}+M_{2}\left(M_{\odot}\right)$ & 2.380 & \pm 0.019 \\
$M_{1} / M_{2}$ & 1.004 & \pm 0.012 \\
$i(\mathrm{deg})$ & 99.4083 & \pm 0.0098 \\
$\omega(\mathrm{deg})$ & 7.735 & \pm 0.013 \\
$\Omega(\mathrm{deg})$ & 23.362 & \pm 0.012 \\
$d(\mathrm{pc})$ & 18.379 & \pm 0.048 \\
$\pi(\mathrm{mas})$ & 54.41 & \pm 0.14 \\
\hline
\end{tabular}

Notes. All parameter uncertainties have been increased by a factor of $\sqrt{\chi_{r}^{2}}=$ 1.09 (though $\chi_{r}^{2}$ of the combined fit is artificial due to rescaling the uncertainties of the individual data sets, this reflects the degree to which the data sets agree with each other). The fit was repeated several times varying the set of nondegenerate parameters used in order to obtain uncertainty estimates for a number of desired quantities. The parameters $\left\{a, R=M_{1} / M_{2}\right\}$ were replaced with the sets $\left\{M=M_{1}+M_{2}, R\right\}$ and $\left\{M_{1}, M_{2}\right\}$. The parallax is a derived quantity.

solar. Absolute magnitudes are also given in Table 3. In each case, the uncertainty in the apparent magnitude $m_{\text {Total }}$ dominates.

\subsection{System Age and Evolutionary Tracks}

The masses and absolute $K$-band magnitudes for the components in this system can be compared to the stellar evolution models from Girardi et al. (2002). As in F2002, an abundance of $Z=0.02$ is assumed. Figure 2 shows the mass versus $K$-band magnitudes for several isochrones downloaded from 
Table 6

Orbital Parameters for $\kappa$ Peg and V819 Her

\begin{tabular}{|c|c|c|c|c|}
\hline \multirow[b]{2}{*}{ Parameter } & \multicolumn{2}{|c|}{$\kappa \mathrm{Peg}$} & \multicolumn{2}{|c|}{ V819 Her } \\
\hline & Value & Uncertainty & Value & Uncertainty \\
\hline$P_{\mathrm{AB}}$ (days) & 4224.76 & \pm 0.74 & 2019.66 & \pm 0.35 \\
\hline$T_{\mathrm{AB}}(\mathrm{MHJD})$ & 52401.52 & \pm 0.96 & 52627.5 & \pm 1.3 \\
\hline$e_{\mathrm{AB}}$ & 0.3140 & \pm 0.0011 & 0.67974 & \pm 0.00066 \\
\hline$i_{\mathrm{AB}}$ (degrees) & 107.911 & \pm 0.029 & 56.40 & \pm 0.13 \\
\hline$\omega_{\mathrm{AB}}$ (degrees) & 124.666 & \pm 0.064 & 222.50 & \pm 0.22 \\
\hline$\Omega_{\mathrm{AB}}$ (degrees) & 289.037 & \pm 0.021 & 141.96 & \pm 0.12 \\
\hline$P_{\mathrm{BaBb}}$ (days) & 5.9714971 & $\pm 1.3 \times 10^{-6}$ & 2.2296330 & $\pm 1.9 \times 10^{-6}$ \\
\hline$T_{\mathrm{BaBb}}(\mathrm{MHJD})$ & 52402.22 & \pm 0.10 & 52627.17 & \pm 0.29 \\
\hline$e_{\mathrm{BaBb}}$ & 0.0073 & \pm 0.0013 & 0.0041 & \pm 0.0033 \\
\hline$i_{\mathrm{BaBb}}$ (degrees) & 125.7 & \pm 5.1 & 80.70 & \pm 0.38 \\
\hline$\omega_{\mathrm{BaBb}}$ (degrees) & 179.0 & \pm 6.0 & 227 & \pm 47 \\
\hline$\Omega_{\mathrm{BaBb}}$ (degrees) & 244.1 & \pm 2.3 & 131.1 & \pm 4.1 \\
\hline$V_{0, \text { Keck }}\left(\mathrm{km} \mathrm{s}^{-1}\right)$ & -9.46 & \pm 0.22 & $\ldots$ & $\ldots$ \\
\hline$V_{1, \text { Keck }}\left(\mathrm{km} \mathrm{s}^{-1} \mathrm{day}^{-1}\right)$ & $-2.2 \times 10^{-4}$ & $\pm 3.4 \times 10^{-4}$ & $\ldots$ & $\cdots$ \\
\hline$V_{2, \text { Keck }}\left(\mathrm{km} \mathrm{s}^{-1}\right.$ day $\left.^{-2}\right)$ & $6.8 \times 10^{-6}$ & $\pm 2.4 \times 10^{-6}$ & $\ldots$ & $\ldots$ \\
\hline$V_{0, \mathrm{C}}\left(\mathrm{kms}^{-1}\right)$ & -9.40 & \pm 0.26 & $\ldots$ & $\ldots$ \\
\hline$V_{0, \text { Lick }}\left(\mathrm{kms}^{-1}\right)$ & -8.37 & \pm 0.26 & $\ldots$ & $\ldots$ \\
\hline$V_{0, M / K}\left(\mathrm{kms}^{-1}\right)$ & $\ldots$ & $\ldots$ & -3.375 & \pm 0.059 \\
\hline$V_{0, \mathrm{DAO}}\left(\mathrm{kms}^{-1}\right)$ & $\ldots$ & $\ldots$ & -3.385 & \pm 0.065 \\
\hline$V_{0, \mathrm{DDO}}\left(\mathrm{kms}^{-1}\right)$ & $\ldots$ & $\ldots$ & -3.35 & \pm 0.12 \\
\hline$M_{\mathrm{A}}\left(M_{\odot}\right)$ & 1.533 & \pm 0.050 & 1.799 & \pm 0.098 \\
\hline$M_{\mathrm{Ba}+\mathrm{Bb}}\left(M_{\odot}\right)$ & 2.472 & \pm 0.078 & 2.560 & \pm 0.067 \\
\hline$M_{\mathrm{Bb}} / M_{\mathrm{Ba}}$ & 0.501 & \pm 0.049 & 0.742 & \pm 0.012 \\
\hline$L_{\mathrm{Bb}} / L_{\mathrm{Ba}}$ & 0.015 & \pm 0.021 & 0.280 & \pm 0.037 \\
\hline$d($ parsecs $)$ & 34.57 & \pm 0.21 & 68.65 & \pm 0.87 \\
\hline$\Phi_{\mathrm{AB}-\mathrm{BaBb}}$ (degrees) & 43.4 & \pm 3.9 & 26.3 & \pm 1.5 \\
\hline$M_{\mathrm{Ba}}\left(M_{\odot}\right)$ & 1.646 & \pm 0.074 & 1.469 & \pm 0.040 \\
\hline$M_{\mathrm{Bb}}\left(M_{\odot}\right)$ & 0.825 & \pm 0.059 & 1.090 & \pm 0.030 \\
\hline$a_{\mathrm{AB}}(\mathrm{AU})$ & 8.122 & \pm 0.063 & 5.108 & \pm 0.046 \\
\hline$a_{\mathrm{BaBb}}(\mathrm{AU})$ & 0.08710 & \pm 0.00091 & 0.04569 & \pm 0.00040 \\
\hline$\pi$ (mas) & 28.93 & \pm 0.18 & 14.57 & \pm 0.19 \\
\hline
\end{tabular}

Notes. Uncertainties for $\kappa$ Pegasi are the maximum of three uncertainties: the uncertainty from the combined fit that included PHASESreweighted data, that including PHASES data with a $161 \mu$ as noise floor, and the difference in the fit values for the two models. The parameters are the average values between a fit including the reweighted uncertainties and one with the noise floor.

http://pleiadi.pd.astro.it and the values derived for the components of $\mu$ Ori. As the most massive component, the properties of Aa provide the strongest constraints on age, being most consistent with isochrones in the age range of $10^{8}-10^{8.5}$ years. This is not entirely consistent with the properties of $\mathrm{Ba}$ and $\mathrm{Bb}$, though close. Of course, if KCTF has played a significant role in the orbital evolution of this system, one wonders whether stellar evolution models for single stars are really applicable to these stars. One would anticipate the evolution of Aa as being affected by tidal forces because it is part of the subsystem near the predicted 140-degree "pile-up." Thus, one would rely more on $\mathrm{Ba}$ and $\mathrm{Bb}$ for system age determination, indicating an age over $10^{9}$ years.

\section{UPDATED ORBITS}

During the course of this investigation, a sign error was found in the analysis software that was used to compute orbital solutions for $\delta$ Equ (Muterspaugh et al. 2005), $\kappa$ Peg (Muterspaugh et al. 2006b), and V819 Her (Muterspaugh et al. 2006a). This error affected fits to the radial velocity data only, with the result that the descending node was misidentified as the ascending, and the angle of periastron passage is off by 180 degrees. Because the software was self-consistent, this has no impact on the mutual inclinations derived. Additionally, the finite travel time of light across the wide orbit is included in the analysis, and this amount was thus incorrect by the same sign error, but the light travel time correction has only a small impact on those models. Both errors have since been corrected.

Twenty-three new observations of $\delta$ Equ and 11 of $\kappa$ Peg have been collected since those initial investigations and are presented in Table 4. Also presented in Table 4 is the complete set of 34 V819 Her PHASES observations with the ten measurements taken during eclipses marked; the previous investigation used less precise methods for predicting eclipse times, so the flagged measurements have changed. Measurements made during eclipse are not used in fitting.

The new analysis makes use of the V819 Her Ba-Bb inclination constraint derived by eclipse lightcurves, a feature not included in the previous study. When computing $\chi^{2}$, an additional term $\left(i_{\mathrm{BaBb}}-i_{\mathrm{BaBb}} \text {, eclipse }\right)^{2} / \sigma_{i, \mathrm{BaBb}}^{2}$, eclipse is added to the sum, where $i_{\mathrm{BaBb} \text {, eclipse }}=80.63$ and $\sigma_{i \text {, BaBb, eclipse }}=0.33 \mathrm{de}$ grees are the value and uncertainty of the $\mathrm{Ba}-\mathrm{Bb}$ inclination from the lightcurve studies of van Hamme et al. (1994). Note that this measurement results from an entirely independent data set. This added constraint lessens covariances between orbital elements.

The corrected and updated orbital solutions are presented in Tables 5 and 6, which are fit to the complete set of PHASES observations and the other astrometric and RV observations tabulated in those previous papers. (A few new measurements 
from speckle interferometry have become available for each system since those investigations. These have little impact on the orbital solutions and are not included in the present fit to avoid overcomplicating this update.) The updated reweighting factors for the PHASES uncertainties for each data set are 3.91 for $\delta \mathrm{Equ}, 7.93$ for $\kappa \mathrm{Peg}$, and 2.0 for V819 Her. Alternatively, the noise floor for $\kappa$ Peg is now found at $161 \mu$ as.

\section{CONCLUSIONS}

The center-of-light astrometric motions of the $\mathrm{Aa}-\mathrm{Ab}$ and $\mathrm{Ba}-\mathrm{Bb}$ subsystems in $\mu$ Ori have been constrained by PHASES observations. While four degenerate orbital solutions exist, two of these can be excluded with high reliability based on massluminosity arguments, and the fact that $\mathrm{Ab}$ is not observed in the spectra. $\mathrm{Ba}$ and $\mathrm{Bb}$ are stars of a class (mid-F dwarfs) whose properties have been well established by studying other binaries. Their association with $\mathrm{Aa}$ and $\mathrm{Ab}$, which are members of more poorly studied classes (Am and late-K dwarfs), allows a better understanding of those objects in a system which can be assumed to be coevolved. The orbital solution finds masses and luminosities for all four components, the basic properties necessary in studying their natures.

Complex dynamics must occur in $\mu$ Ori. The $\mathrm{Ba}-\mathrm{Bb}$ orbital plane is nearly perpendicular to that of the A-B motion, and certainly undergoes Kozai-type inclination-eccentricity oscillations. It is possible that the mutual inclination of the A-B pair and $\mathrm{Aa}-\mathrm{Ab}$ subsystem is a result of KCTF effects over the system's evolution.

Finally, it is noted that the orbits in the $\mu$ Ori system are quite non-coplanar. This is in striking contrast with the planets of the solar system, but follows the trend seen in triple star systems. With the solar system being the only one whose coplanarity has been evaluated, it is difficult to draw conclusions about the configurations of planetary systems in general. It is important that future investigations evaluate the coplanarities of extrasolar planetary systems to establish a distribution. Whether that distribution will be the same as or different from that of their stellar counterparts may point to similarities or differences in star and planet formation, and provide a key constraint on modeling multiple star and planet formation.

We thank Daniel Fabrycky for helpful correspondence about his recent theoretical work on KCTF. We thank Bill Hartkopf for providing weights for the new non-PHASES differential astrometry measurements. PHASES benefits from the efforts of the PTI collaboration members who have each contributed to the development of an extremely reliable observational instrument. Without this outstanding engineering effort to produce a solid foundation, advanced phase-referencing techniques would not have been possible. We thank PTI's night assistant Kevin Rykoski for his efforts to maintain PTI in excellent condition and operating PTI in phase-referencing mode every week. Part of the work described in this paper was performed at the Jet Propulsion Laboratory under contract with the National Aeronautics and Space Administration. Interferometer data were obtained at the Palomar Observatory with the NASA Palomar Testbed Interferometer, supported by NASA contracts to the Jet Propulsion Laboratory. This publication makes use of data products from the Two Micron All Sky Survey, which is a joint project of the University of Massachusetts and the Infrared Processing and Analysis Center/California Institute of Technology, funded by the National Aeronautics and Space Administration and the National Science Foundation. This research has made use of the Simbad database, operated at CDS, Strasbourg, France. MWM acknowledges support from the Townes Fellowship Program. PHASES is funded in part by the California Institute of Technology Astronomy Department, and by the National Aeronautics and Space Administration under grant no. NNG05GJ58G issued through the Terrestrial Planet Finder Foundation Science Program. This work was supported in part by the National Science Foundation through grants AST 0300096, AST 0507590, and AST 0505366. The work of F.C.F. has been supported in part by NASA grant NCC5-511 and NSF grant HRD-9706268. M.K. is supported by the Polish Ministry of Science and Higher Education through grants N203 005 32/0449 and 1P03D 02129.

\section{REFERENCES}

Aitken, R. G. 1914, One hundred new double stars: twenty-second list, Lick Obs. Bull., 8, 93-95

Armstrong, J. T., et al. 1998, The Navy Prototype Optical Interferometer, ApJ, 496, 550-571

Colavita, M. M., et al. 1999, The Palomar Testbed Interferometer, ApJ, $510,505-521$

Duquennoy, A. 1987, A study of multiple stellar systems with CORAVEL, A\&A, 178, 114-130

Eggleton, P. P., \& Kiseleva-Eggleton, L. 2001, Orbital evolution in binary and triple stars, with an application to SS Lacertae, ApJ, 562, 1012-1030

Fabricius, C., Høg, E., Makarov, V. V., Mason, B. D., Wycoff, G. L., \& Urban, S. E. 2002, The Tycho double star catalogue, A\&A, 384, 180-189

Fabrycky, D., \& Tremaine, S. 2007, Shrinking binary and planetary orbits by Kozai cycles with tidal friction, ApJ, 669, 1298

Fekel, F. C. 1980, The close multiple system MU Orionis-masses and the mass-luminosity relation, PASP, 92, 785-789

Fekel, F. C., Scarfe, C. D., Barlow, D. J., Hartkopf, W. I., Mason, B. D., \& McAlister, H. A. 2002, The quadruple system $\mu$ Orionis: three-dimensional orbit and physical parameters, AJ, 123, 1723-1740

Frost, E. B. 1906, Spectrographic observations. Four stars with variable radial velocities, ApJ, 23, 264-269

Girardi, L., Bertelli, G., Bressan, A., Chiosi, C., Groenewegen, M. A. T., Marigo, P., Salasnich, B., \& Weiss, A. 2002, Theoretical isochrones in several photometric systems. I. Johnson-Cousins-Glass, HST/WFPC2, HST/NICMOS, Washington, and ESO Imaging Survey filter sets, A\&A, 391, 195-212

Heintz, W. D. 1996, A study of multiple-star systems, AJ, 111, 408-441

Horch, E. P., Robinson, S. E., Meyer, R. D., van Altena, W. F., Ninkov, Z., \& Piterman, A. 2002, Speckle observations of binary stars with the WIYN Telescope. II. Relative astrometry measures during 1998-2000, AJ, 123, 3442-3459

Horch, E., van Altena, W. F., Girard, T. M., Franz, O. G., López, C. E., \& Timothy, J. G. 2001, Speckle interferometry of southern double stars. II. Measures from the CASLEO 2.15 meter telescope, 1995-1996, AJ, 121, 1597-1606

Hummel, C. A., et al. 2003, First observations with a co-phased six-station optical long-baseline array: application to the triple star $\eta$ virginis, AJ, $125,2630-2644$

Kozai, Y. 1962, Secular perturbations of asteroids with high inclination and eccentricity, AJ, 67, 591-598

Lane, B. F., \& Colavita, M. M. 2003, Phase-referenced stellar interferometry at the Palomar Testbed Interferometer, AJ, 125, 1623-1628

Lane, B. F., \& Muterspaugh, M. W. 2004, Differential astrometry of subarcsecond scale binaries at the Palomar Testbed Interferometer, ApJ, $601,1129-1135$

Lane, B. F., et al. 2007, The orbits of the quadruple star system 88 tau a from phases differential astrometry and radial velocity, ApJ, 669, 1209

Lestrade, J., Phillips, R. B., Hodges, M. W., \& Preston, R. A. 1993, VLBI astrometric identification of the radio emitting region in Algol and determination of the orientation of the close binary, ApJ, 410, 808-814

Muterspaugh, M. W., Lane, B. F., Konacki, M., Burke, B. F., Colavita, M. M., Kulkarni, S. R., \& Shao, M. 2005, PHASES high-precision differential astrometry of $\delta$ Equulei, AJ, 130, 2866-2875

Muterspaugh, M. W., Lane, B. F., Konacki, M., Burke, B. F., Colavita, M. M., Kulkarni, S. R., \& Shao, M. 2006a, PHASES differential astrometry and 
the mutual inclination of the V819 Herculis triple star system, A\&A, 446, 723-732

Muterspaugh, M. W., Lane, B. F., Konacki, M., Wiktorowicz, S., Burke, B. F., Colavita, M. M., Kulkarni, S. R., \& Shao, M. 2006b, PHASES differential astrometry and iodine cell radial velocities of the $\kappa$ Pegasi triple star system, ApJ, 636, 1020-1032

Muterspaugh, M. W., Lane, B. F., Kulkarni, S. R., Burke, B. F., Colavita, M. M., \& Shao, M. 2006c, Limits to tertiary astrometric companions in binary systems, ApJ, 653, 1469-1479

Orlov, V. V., \& Petrova, A. V. 2000, Dynamical stability of triple stars, Astron. Lett., 26, 250-260

Orlov, V. V., \& Zhuchkov, R. Y. 2005, Analysis of the dynamic stability of selected multiple stars with weak hierarchy, Astron. Rep., 49, 201-216

Pan, X., Shao, M., \& Colavita, M. M. 1993, High angular resolution measurements of Algol, ApJ, 413, L129-L131
Perryman, M. A. C., et al. 1997, The HIPPARCOS catalogue, A\&A, 323, L49-L52

Scardia, M. 2007, Speckle observations with PISCO in Merate. III. Astrometric measurements of visual binaries in 2005 and scale calibration with a grating mask, MNRAS, 374, 965-978

Skrutskie, M. F., et al. 2006, The Two Micron All Sky Survey (2MASS), AJ, $131,1163-1183$

Smith, R. C. 1983, An empirical stellar mass-luminosity relationship, The Observatory, 103, 29-31

Söderhjelm, S. 1999, Visual binary orbits and masses post Hipparcos, A\&A, $341,121-140$

Sterzik, M. F., \& Tokovinin, A. A. 2002, Relative orientation of orbits in triple stars, A\&A, 384, 1030-1037

van Hamme, W. V., et al. 1994, The two variables in the triple system HR $6469=$ V819 Her: one eclipsing, one spotted, AJ, 107, 1521-1528 